\title{
More self-organization, more control-or even both? Inverse transparency as a digital leadership concept
}

\author{
Maren Gierlich-Joas ${ }^{1}$ (D) Thomas Hess ${ }^{1}$ • \\ Rahild Neuburger ${ }^{2}$
}

Received: 15 October 2019/Accepted: 15 October 2020/Published online: 29 October 2020

(C) The Author(s) 2020

\begin{abstract}
Digital innovations drive an organization's digital transformation. While numerous studies focus on digital product and service innovation, digital process innovation and novel business models, management and leadership concepts are primarily investigated as enabling framing conditions in previous contributions. However, management and leadership concepts have changed dramatically in the digital era. The rise of digital technologies has led to companies acquiring large amounts of data. Moreover, novel technical solutions facilitate the analysis and processing of this data, leading to an increase in organizational transparency. Traditional leadership theories fail to explain the influence of digitalization and increasing transparency of leadership. In a digitized world, managers often face a trade-off when using data for management purposes. On the one hand, transparency leads to decreasing information asymmetries, allowing managers to monitor employees' actions at low cost. On the other hand, employees demand self-organization and empowerment. In this context, new forms of control and employee engagement need to be designed. With our conceptual paper, we aim to provide a solution to the challenges of using transparency in leadership in a mutually beneficial way for managers and employees by introducing the concept of "inverse transparency." We develop the concept building on the existing literature on
\end{abstract}

Maren Gierlich-Joas

gierlich@bwl.lmu.de

Thomas Hess

thess@bwl.lmu.de

Rahild Neuburger

neuburger@bwl.lmu.de

1 Institute for Information Systems and New Media, LMU, Munich School of Management, Munich, Germany

2 Institute for Information, Organization and Management, LMU, Munich School of Management, Munich, Germany 
transparency and leadership. We see inverse transparency as the basis for a new type of digital innovation, which we introduce as digital leadership innovation. Thus, we enhance current research on leadership approaches and digital innovation and create a theoretical basis for further research.

Keywords Digital innovation · Digital transformation - Leadership · Transparency $\cdot$ Empowerment $\cdot$ Inverse transparency

\section{Introduction}

In the digital area, the impact of digital transformation on markets and organizations has become clearly visible (Sebastian et al. 2017). Driven by the availability of data and digital technologies, firms innovate their processes, business models, products and services (Nambisan et al. 2017). This development is accelerated by continuous innovations in the field of information and communication technology. With the decreasing costs of communication tools, the widespread use of the internet and expanding options for data handling, new ways of collaboration, raising, storing and transmitting data have become feasible (Châlons and Dufft 2017). The technologies used are not stand-alone systems but contribute to an interconnected landscape of information systems (IS) that initiate new opportunities for digital transformation (Henfridsson and Bygstad 2013).

While numerous studies focus on digitization, implying changes on a processual level with the goal of increasing efficiency and cutting costs, those that focus on digital transformation investigate changes in business models and organizational structures (Parviainen et al. 2017). Digital transformation entails "the combined effects of several digital innovations, bringing about novel actors (and actor constellations), structures, practices, values, and beliefs that change, threaten, replace or complement existing rules of the game within organizations and fields" (Hinings et al. 2018, p. 53). In this context, digital innovations are an essential part of an organization's digital transformation (Wiesböck and Hess 2020).

A digital innovation can be defined as two digital artifacts: an innovative digital solution and an innovative digital business concept (Wiesböck and Hess 2020). The digital solution entails the technical side of a digital innovation that consists of an IT artifact, whereas the innovative digital business concept addresses the organizational dimension and new business requirements (Kohli and Melville 2018; Wiesböck and Hess 2020). The success of digital innovations depends on the balanced integration of both artifacts. Digital innovations can be conceptualized in three ways: new digital products and services, new digital processes and new digital business models (Nambisan et al. 2017; Wiesböck and Hess 2020). In the logic of digital innovation, organizational structures such as culture or leadership are often considered as enabling framing conditions for digital innovations (Wiesböck and Hess 2020). However, innovative digital leadership concepts can be seen not only as enablers but also as the core of digital innovations.

As we argue in this paper, the concept of digital innovations is applicable to innovative digital leadership concepts (see Fig. 1). Analogous to the logic illustrated 


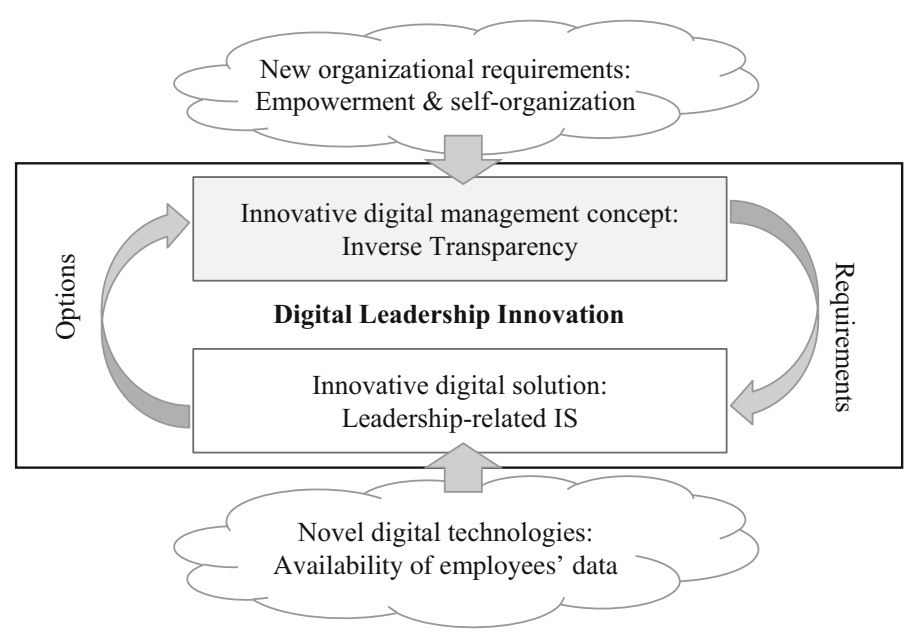

Fig. 1 Technology push-pull model of digital leadership innovations (adapted from Wiesböck and Hess 2020)

in Fig. 1, digital innovations in the field of leadership are triggered by a technology push. The generation of data on employees occurs as a by-product of data-driven organizations. As data become available at little cost and in high volumes, it becomes easier for managers to track employee behavior in great detail (Tursunbayeva et al. 2018). Information systems assist in storing, analyzing and communicating these data. Since the 1950s, IS in the area of human resources (HR) have expanded drastically. Ranging from basic payroll systems to advanced management IS and more sophisticated people analytics, the set of functions of leadership-related IS has increased significantly (Johnson et al. 2016a; Tursunbayeva et al. 2018). These specialized tools, which are driven by the availability of employee data, represent the innovative digital solution of the digital innovation.

Many firms have already implemented the technical artifacts (Khatri and Samuel 2019), but the organizational side needs to be innovated as well. Next to new digital solutions, organizational requirements are also changing; employees are asking for more empowerment and participation (Carasco-Saul et al. 2015; Hays et al. 2017). These developments-technology push and technology pull-are the drivers for new innovative leadership concepts that cover the organizational artifact of digital innovations. New types of leadership are investigated (Avolio et al. 2014) and new capabilities of leaders are defined (Cortellazzo et al. 2019). Thus, innovative digital leadership concepts can be understood as a digital innovation. This new supportive type of digital innovation, which we call "digital leadership innovation," can enhance the established types of digital innovations (see Fig. 1).

While much research focuses on the three levels novel digital technologies, innovative digital solutions and new organizational requirements (see Fig. 1), the fourth layer, innovative digital leadership concepts, remains under studied. In studies of digital leadership in the field of management, various aspects of leadership in a digital world are analyzed, but "in a fragmented fashion across 
various disciplines" (Cortellazzo et al. 2019, p. 2). Some studies point out that a combination of people-oriented and technical skills is essential for successful e-leadership (Diamante and London 2002; Henttonen et al. 2012). The new C-level roles of CIOs and CDOs have also received attention (Avolio et al. 2000; Cortellazzo et al. 2019). Particular attention has been paid to the adoption of digital tools by leaders and their distribution in firms (Haddud and McAllen 2018). New leadership forms for virtual teams have also been examined (Hambley et al. 2007). In the field of IS, research focuses on the technological drivers of new leadership approaches (Gal et al. 2017; Johnson et al. 2016b) and the governance of digital transformation processes (Bharadwaj et al. 2013). Finally, organizational roles and transformation strategies, rather than digital leadership approaches, have been studied (Tumbas et al. 2018; Vial 2019). Thus, an in-depth analysis of digital innovations in the field of leadership, especially digital management concepts, is currently lacking.

Transparency plays a crucial role in digital leadership innovations. The expanding volume of data available combined with powerful new technologies leads to higher overall transparency in companies and strongly influences leadership (Cortellazzo et al. 2019; Tursunbayeva et al. 2018). From an ethical point of view, the increasing amount of employee data available poses challenges for leaders. On the one hand, leaders need to manage new control mechanisms (Wiener et al. 2019) and on the other, transparency can be used to empower employees and to make internal processes visible, stressing the positive effects of transparency (Heuman et al. 2015). Is it true that the more transparency, the better? This assumption is not valid when implementing transparency in leadership. Managers and employees find themselves on different sides of the field of tension, as they have to balance the positives and negatives of transparency in leadership. We offer a solution for this tension and strive to "go beyond traditional leadership theories to explain the impact digitalization exerts on leadership and leaders" (Cortellazzo et al. 2019, p. 15). Thus, we propose the following research questions:

RQ 1 How did the concepts of transparency and leadership develop in the context of digitalization?

RQ 2 How can transparency in leadership be integrated and implemented so that it results in an innovative digital leadership concept?

In pursuit of the first research question, we will provide an overview of current research on leadership and transparency in a digitized world. Building on the results of this overview, we introduce an innovative digital leadership concept for integrating transparency into leadership. We follow the logic of digital innovations and address the organizational artifact.

Our paper consists of the following major sections. Following the introduction, we highlight the theoretical concepts of leadership and transparency, and then discuss the influence of digitalization on both concepts in the second section. In section three, we outline the impact of transparency on leadership in light of agency theory and identify the tensions that arise when implementing transparency in leadership. To address these tensions, we introduce the concept of inverse 
transparency as the basis for digital leadership innovation. With this approach, we aim to overcome obstacles to transparent leadership by outlining its benefits for all stakeholders. We analyze the theoretical concept through the lens of control and outline a conceptual implementation process in section four. Finally, we highlight the implications of our research, its limitations and directions for future research.

\section{Theoretical concepts}

\subsection{Leadership}

Digitalization's effect on leadership is a topic of great interest to IS and HR. To create a common basis, we first outline our understanding of leadership, drawing on multiple disciplines. Next, we focus on the impact of digitalization on leadership from the perspective of IS.

\subsubsection{Understandings of leadership}

The concept of leadership has a long history and is deeply rooted in the field of management. Some definitions focus on leadership as a process in which a manager intentionally influences other people (Yukl 2013). Others describe specific leadership functions, such as the interpersonal, the information processing and the decision-making role (Mintzberg 2007). Having reviewed multiple definitions, we define leadership as the management of employee relations and the exercise of authority to co-ordinate tasks within a company to fulfill operative and strategic goals (Beare et al. 1997). This definition has been regularly employed in recent scholarship (Korica et al. 2015), and from our perspective, it combines the interpersonal, decision-making and information processing roles of leaders well.

In addition to scholarship on the dimensions and definitions of leadership, a rich body of literature exists on leadership theories and leadership concepts. Leadership theories offer explanations for leadership decisions or forecast future developments, and in turn, leadership concepts draw on these abstract theories to tackle the implementation of concrete guidelines in practice (Lang and Rybnikova 2014).

Leadership theories and concepts have changed significantly over the years. In the 1920s, theories stressed the role of leaders and their personalities, but in subsequent years, the theories have shifted to a focus on the contextual factors and the personality of the follower. More recently, concepts of leadership have taken external factors, such as the availability of technology, into account (Dinh et al. 2014).

\subsubsection{Leadership and digitalization from a management perspective}

Because of the interdisciplinary nature of the topic, business, management, accounting, psychology and social science have all addressed the interplay of digitalization and leadership, taking either a macro or a micro perspective on the topic (Cortellazzo et al. 2019). On the macro level, e-leaders and new organizational 
forms (Henttonen et al. 2012), tools that facilitate leadership and how to implement them (Cortellazzo et al. 2019) and ethical challenges for leaders have been discussed. The need to balance privacy protection and controlling mechanisms in times of nearly unrestricted availability of data has also received attention (Hofmann 2007).

On the micro level, scholars have focused on the evolution of C-level roles, leaders' skills in the digital era and virtual teams (Cortellazzo et al. 2019). The importance of CIOs and their ability to create a competitive IT infrastructure is increasing, forcing CEOs and CIOs to interact closely (Gerth and Peppard 2016). The combination of people-orientated and technical skills is considered a key factor for digital leaders (Diamante and London 2002), and leaders are required to develop intercultural competencies as they interact with many cultures (Schwarzmüller et al. 2018). Lastly, virtual teams are of great scientific interest. Because of the availability of collaboration tools, individuals in different time zones and spaces and within various organizational boundaries can work together in a decentralized team (Hambley et al. 2007).

Overall, the different disciplines offer heterogeneous perspectives on leadership, ranging from technology-related approaches to ethical questions and individual requirements for leaders. However, the influence of digitalization on leadership remains understudied. In the following subsection, we analyze how IS tackles this research gap.

\subsubsection{Leadership and digitalization from the perspective of information systems}

Driven by the increasing influence of technology on leadership, a growing body of IS literature has developed that draws on well-established HR studies, but also takes new directions. Three major research streams on leadership and digitalization in the field of IS can be identified. The first one is the technological perspective on leadership-related IS. Enabled by the availability of data, a new area of IS has emerged in the field of human resource management (HRM), expanding the range of functionalities of former enterprise resource planning systems (ERP) (Johnson et al. 2016b). In this context, human resource information systems (HRIS) can be defined as "system[s] used to acquire, store, manipulate, analyze, retrieve, and distribute information regarding an organization's human resources to support HRM and managerial decisions" (Kavanagh and Johnson 2018, p. 8). Distinct types of HRIS, like people analytics, have been investigated, and research interest in all fields has continually been growing during the last ten years (Tursunbayeva et al. 2018). A large body of research on the adoption of these tools (Maier et al. 2013), their areas of application and their organizational impact exists ( $\mathrm{Gal}$ et al. 2017). Because of technological advances, the transparency and efficiency of HRM processes is increasing. Tasks, ranging from operative recruiting activities to strategic leadership aspects, are supported by leadership-related IS (Gal et al. 2017).

A second major research stream tackles strategic organization. Studies shed light on the governing of digital transformation processes in companies (Bharadwaj et al. 2013), and digital transformation strategies provide guidance and emphasis the need to consider each dimension of the company (Matt et al. 2015). Furthermore, the role 
of the CDO and its distinction from the former role of the CIO is an essential research topic (Haffke et al. 2016; Tumbas et al. 2018). This research stream offers valuable insights into the strategic implementation and leadership of digital transformation. However, implications for leadership concepts cannot be derived from this literature.

The final research stream consists of literature on digital leadership approaches from an IS perspective. It touches on the organizational side of digital innovation by addressing innovative digital leadership concepts. One example being agile work, which is widely used in software development and which affects the interaction between manager and employee (Abrahamsson et al. 2017). Because of dynamic working structures and the empowerment of employees, managers retain less power and primarily use collaborative leadership styles like transformational leadership (Nerur et al. 2005). Leadership styles are changing because of the availability of data, and managers make decisions in more transparent ways (Gierlich and Hess 2020). A similar effect can be observed in e-leadership and digital leadership (Avolio et al. 2000). IS scholars contribute to literature on e-leadership as "[it] takes shape in a virtual context where collaboration and leader-follower interaction are mediated by ICT" (Haffke et al. 2016, p. 188).

Finally, IS research focuses on the technological drivers for new leadership approaches, the leadership of digital transformation and on new leadership concepts enabled by new technologies. Overall, the technological side of digital innovation is present in IS literature, whereas the organizational side, represented by innovative digital leadership approaches, remains largely untouched. Some topics closely relate to the research discipline of management. Despite the rising interest in the influence of digitalization on leadership, innovative digital leadership concepts receive attention mainly in management and HR.

\subsection{Transparency}

\subsubsection{Understanding of transparency}

Transparency is closely related to the concept of leadership and has been a topic of researchers for many years. However, the understanding of what transparency is has changed over time. The earliest studies emerged in the field of philosophy with a focus on the term "observations" before the concept was adopted in natural science and later in management science (Bernstein 2017). Especially in the 1970s and 1980s, research on transparency grew in importance (Bernstein 2017).

Transparency has been defined differently in the disciplines of sociology, psychology, management and IS (Bernstein 2017). In some definitions, transparency is seen as monitoring, a "non-hierarchical observation system that gathers information about an activity or a task and makes it widely available" (Bernstein 2017 , p. 218). This form of transparency is reported to positively influence employees' motivation, their empowerment and learning (Christensen and Cheney 2015). But when monitoring is implemented in the hierarchical relationship between managers and employees, transparency is seen as surveillance (Bernstein 2017). This is considered to have ambiguous effects on the relationship between employee 
and manager, either increasing compliance and productivity or decreasing motivation (Pierce et al. 2015).

Other studies build on a more neutral view of process transparency, "the comprehensibility of the [...] process" for the observer (Nussbaumer and Schwabe 2012 , p. 4). In other words, the focus of observation is on the workflow and not on individuals (Bernstein 2017). Last but not least, transparency has been described as the frequency of information disclosure (Berglund 2014): "information must be openly shared for it to be considered transparent" (Schnackenberg and Tomlinson 2016, p. 1792). The perspective of transparency as disclosure is common in IS research, especially in research on privacy. Next to the quantity of information transmitted, quality is essential (Albu and Flyverbom 2019). In this work, we define transparency as the disclosure of information among different groups of people within organizations to enhance trust and accountability.

\subsubsection{Design choice for transparency in companies}

An individual's perception of transparency is closely related to its implemented scope, its design and its perceived utility. Hence, the design of transparency tools affects their later adoption. Often, design choices are made between different characteristics of transparency, influencing the dimensions what?, when? and how?

The most crucial question is "What object, process or data is transparent?" Transparency can refer to data or to physical environments (Bernstein 2017). Moreover, outcome and process transparency can be differentiated (Nussbaumer and Schwabe 2012). Making a process transparent results in greater empowerment of employees but it raises privacy concerns. In contrast, outcome transparency is preferred by employees, but it is less insightful regarding the understandability of a process (Schnackenberg and Tomlinson 2016). In this context, the level of analysis is important, and aggregated and anonymous data is preferred over data that reveals identity (Schnackenberg and Tomlinson 2016).

The second important question is "When and for how long is transparency generated?" Permanent transparency is considered to be a greater threat than temporary transparency (Schnackenberg and Tomlinson 2016). In the working context, an example of permanent transparency is the observation of employees via video, whereas monthly performance reports of employees would be considered temporary transparency. Furthermore, when data is visible plays a crucial role. A prompt transmission of data increases employees' penchant to keep details about their performance from their leader, while a delayed form of transparency is perceived as less critical (Schnackenberg and Tomlinson 2016).

A third important design choice to be addressed is "At what organizational level and in which direction is transparency generated?" Within organizations, transparency can be generated upwards, which makes it possible for managers to observe employees' behavior, which is crucial to monitoring (Heald 2006). In contrast, transparency can also be implemented downwards. This means that employees can observe the conduct and behavior of managers (Heald 2006). Finally, individuals' option for consent-based transparency is highly valued instead of being restricted to mandated transparency (Gerlach et al. 2015). These design 
choices illustrate the importance of the design process of transparency in companies and its potential impact on leadership.

\subsubsection{Transparency and digitalization}

Like leadership, transparency is influenced by digitalization. The interplay between the two concepts is outlined in the following section. Two effective directions of transparency can be distinguished. First, transparency is enabled by technologies. Second, transparency sets the framing conditions for the use of technologies. It enables new ways for collaboration and leadership, which would be difficult to implement without transparency.

For the first direction, numerous studies focus on technologies generating data and enabling higher levels of transparency in firms (Gierlich and Hess 2020). During the last few years, the availability of data has increased dramatically in terms of volume, value, variety and velocity (Wamba et al. 2015). These data stem from multiple sources internal and external to companies. For example, in the supply chain, goods are equipped with sensors, localizing their precise position and status (Aung et al. 2014). Identification technologies like bar codes and RFID, internet applications or web technologies are widely established as innovative digital solutions in many firms (Aung et al. 2014). On the production level, physical devices are connected by sensors, GPS or RFID tags (Mittal et al. 2019). In these cyber-physical-systems (CPS), a lot of data are raised and exchanged in real time, so the quality of production is improved as scrap rates lessen (Mittal et al. 2019). When using CPS or identification technologies, employee data collection is a by-product.

Moreover, new technologies that overtly collect data on employees have evolved. As technologies in the field of HRM have grown from basic payroll systems to advanced ERP systems and modern HRIS, more employee data are being collected (Johnson et al. 2016b). A wide set of operational data like employees' working hours, breaks taken and hourly efficiency can now be documented automatically (Gal et al. 2017). In specific industries, for example, logistics or customer support, the employees' picking efficiency in warehouses or their performance answering calls can be monitored (Backhaus 2019).

In addition to the availability of data increasing, the functionalities of big data technologies have expanded drastically so that data can be processed properly (Rodríguez-Mazahua et al. 2016). Looking at machine learning, data mining, signal processing and visualization techniques, the number and quality of big data tools have increased enormously. These tools assist in drawing insights from datasets and interpreting them (Rodríguez-Mazahua et al. 2016) and in predicting future developments, for example, market trends or the fluctuation of employees (Gal et al. 2017). Thus, they avoid information overload caused by vast amounts of unstructured data (Edmunds and Morris 2000). In sum, the combination of data's availability and suitable technologies for their analysis drives transparency in the different organizational levels (see Fig. 2).

However, analyzing the interplay of digitalization and transparency from another perspective, transparency is an important basis for the use of digital technologies. Transparency influences a firm's culture and strategy. On the level of business 


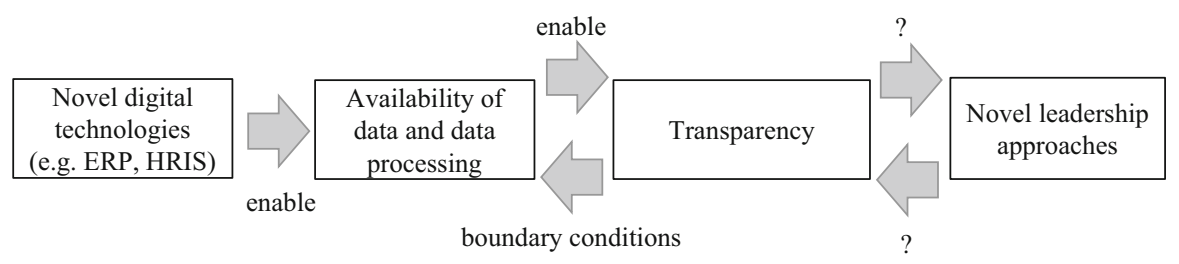

Fig. 2 The interplay between technology and transparency

models, transparency can become the core value proposition and strategic competence of a firm. A transparency strategy helps make information an important component of competition in a digital world (Granados and Gupta 2013). On the level of collaboration, transparency helps to empower employees and to establish a culture of trust (Albu and Flyverbom 2019). Only if a suitable level of transparency is implemented can technologies be used to their full extent. Otherwise, the collection and analysis of data is restricted. Hence, transparency is a framing condition for data's availability and usage (see Fig. 2).

Finally, transparency has been well researched in IS. Studies on the interplay of transparency and digitalization highlight the close connection between the two. This study will further focus on the relation between transparency and leadership, which has been only minimally studied.

\section{The impact of transparency on leadership: tensions and conceptual solution}

\subsection{Interpreting the interplay between transparency and leadership using agency theory}

Having described the status quo for the concepts of leadership and transparency, we now turn to analyzing the interplay between the two. Previously, scholars have outlined the interdependencies of transparency and leadership in light of different theories, for example, advanced information technologies theory, organizational information processing theory, adaptive structuration theory and agency theory (Cortellazzo et al. 2019). In our study, we focus on leadership as the relation between manager and employee and thus consider agency theory as most suitable for our analysis.

Agency theory has been widely applied in the disciplines of accounting, economics, organizational behavior, sociology and IS (Eisenhardt 1989). In IS, agency theory is especially used in the area of IS development, knowledge management and organizational performance (Lim et al. 2013). It sheds light on the relationship between employee and employer and the influence of transparency from a conceptual point of view. "Agency theory is concerned with resolving two problems that can occur in agency relationships. The first is the agency problem that arises when (a) the desires or goals of the principal and agent conflict and (b) it is difficult or expensive for the principal to verify what the agent is actually doing" 
(Eisenhardt 1989, p. 58). Agency theory helps in understanding the origin and the difficulties of information asymmetries between both stakeholders.

To reduce information asymmetries, two strategies have traditionally been applied: principals use screening mechanisms to monitor their agents' activities and agents invest in signaling activities to reveal their behavior to the principal (Aral et al. 2012) (see Fig. 3). Signaling and screening lead to agency costs and inefficiencies on the part of the principal and the agent (Picot 1989). Therefore, a reduction of information asymmetries, resulting in a decrease in agency costs, is highly desirable (Eisenhardt 1989). The crucial question is how to use the data in a sensible way.

An expanding amount of data has a significant impact on the interpretation of agency theory (de Camargo Fiorini et al. 2018). Today, information can easily be spread among different actors, reducing information asymmetries and agency costs (de Camargo Fiorini et al. 2018). Starting with the hypothesis that increased transparency can lower agency costs in leadership relations, one could argue that transparency holds a maximization function: the more transparency, the better. However, studies highlight potential fields of tension when leadership is made transparent. The trade-offs are discussed individually in the following paragraphs.

\subsubsection{Fields of tension from a manager's perspective}

From the managerial point of view, the increasing availability of data and the resulting transparency result in certain challenges. Because of diminishing control costs, it gets easier for managers to track their employees on a granular level (Hess 2002), and information asymmetries between agent and principal decrease drastically. Consequently, hierarchical leadership can be put in place, driven by the desire for total control. This type of leadership results in highly centralized processes and mistrust. In the 1980s and 1990s, performance measurements based

Fig. 3 Interpreting leadership and transparency using agency theory

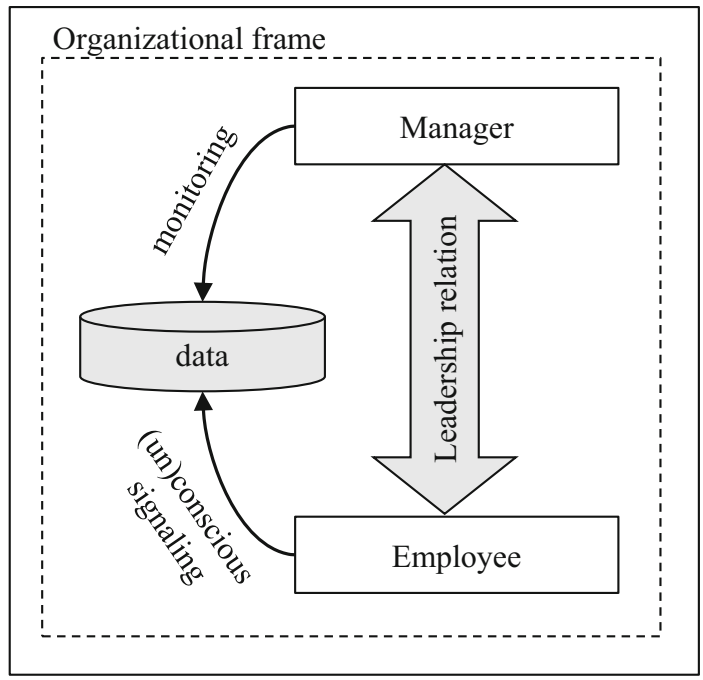


on easily quantifiable work were used (Stanton and Weiss 2000). But more recently, complex work-related activities, such as website visits or social interactions among employees, are traced (Stanton and Weiss 2000). Most of the studies identify the negative influence of strict control mechanisms on employee satisfaction and performance.

In contrast, transparency can also be implemented in employee-centric leadership approaches that are driven by empowerment and embrace the changing values of employees. Surveys stress the demand for higher levels of participation and selfrealization on the part of employees (Hays et al. 2017). Today, it is seen as important that leadership addresses the needs of the employees (Dinh et al. 2014). Still, the risks and opportunities of using transparency-based leadership leads to a dilemma for managers in their everyday decision-making.

\subsubsection{Tension from the employees' perspective}

Employees experience tension between positively and negatively perceived transparency. On the one hand, transparency can be seen positively as a performance incentive, leading to increased motivation, cooperation and willingness to compromise (Hofmann 2007). Studies indicate that employees benefit from transparent leadership approaches in a variety of ways. As their performance can be closely monitored, appropriate compensation and performance-based bonuses can be given (Boudreau and Cascio 2017). Though not yet fully implemented, transparency might be able to redefine firms' compensation schemes in the future. Employees can be rewarded for their performance, not only on a monetary level but also on an intrinsic level, for example, individual training sessions for employees to improve their personal development can lead to higher retention rates (Gal et al. 2017). Moreover, if employees gain comprehensive knowledge of the processes they are involved in, they are empowered to be more self-organized and responsible in their work (Carasco-Saul et al. 2015). In sum, these aspects lead to higher job satisfaction and increased levels of performance.

But studies also indicate that there are multiple risks for employees. When transparency is not interpreted as disclosure or objective monitoring, but as surveillance by one hierarchical level of another, employees' trust in their organization decreases (Alder 2001). If transparency is used for finger-pointing and laying blame instead of for constructive improvement, undesirable effects may follow, for example, a decrease in employee motivation (Valentinov et al. 2019). Finally, transparency perceived negatively can lead to employee stress and health issues (Alder 2001). The use of cameras at cash desks serves as an example of both aspects of transparency. Cameras are a form of protection against robbery, but they can also be misused for monitoring an employee's efficiency. Thus, if leadership is to be constructive, the tension between the positive and negative effects of transparency for the employee must be carefully balanced. The perception of transparency from the employees' point of view depends on the way that transparency is introduced. The use of aggregated data, confidential treatment of the data, consent-based decision-making and incentives for the employee to assist in 
shaping a positive form of transparency in organizations are all ways of using transparency effectively (Bernstein 2017; Schnackenberg and Tomlinson 2016).

\subsection{Inverse transparency as a solution to the tensions between manager and employee}

In this section, we conceptualize the basis for the digital management innovation layer of the digital innovation, which we call inverse transparency. The concept aims to overcome the trade-offs that agency theory exposes. Inverse transparency was first mentioned in Brin's 1998 novel The transparent society, in which the author describes a futuristic city where every action is being monitored by the police. In a second scenario, the citizens themselves are empowered to oversee the police, which Brin described as "inverse transparent" (Brin 1998).

In what follows, we define inverse transparency as the empowerment of employees by making the flow and the usage of data transparent within organizations (Boes 2020). As an initial step, the flow of data should be visible. Employees should be aware of data been collected about themselves and their performance. The type of data, the level of anonymization and aggregation use, as well as data storage play a crucial role in the privacy decisions of employees. Second, employees should not only be informed about the collection of data but also about how the data is analyzed, distributed and made accessible; the traceability of data is the basis for transparency (see Fig. 4).

What distinguishes inverse transparency from the traditional definition of transparency is the degree of employee participation. Employees are expected to be an active part of the firm's data protection mechanism. The individual should be able to control the collection, accessibility and further usage of data about themselves. The principle of consent-based decision-making about an individual's data is essential for inverse transparency. Employees should be able to access all

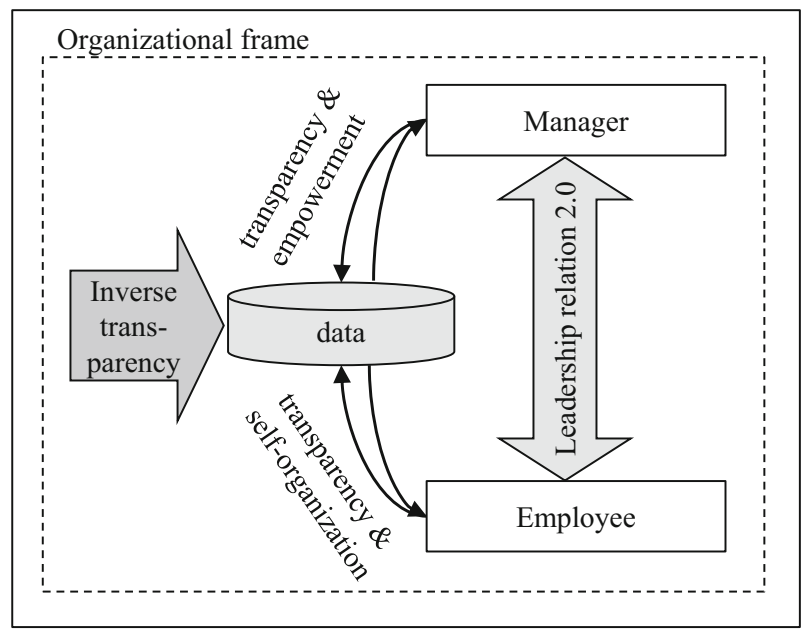

Fig. 4 New leadership relationships based on inverse transparency 
data collected about themselves so that they gain a holistic overview about the organization and their daily tasks. With this knowledge, they are empowered to improve processes and work in a more self-organized and efficient way. If employees are motivated by inverse transparency, they are more likely to act in way that will a benefit the entire company. At that stage, supportive and empowering leadership approaches, rather than regulative ones, are the most helpful. Hence, inverse transparency does not only address employee well-being, it also has positive implications for managers and their leadership approaches.

Positioning inverse transparency in the research field of transparency, we build on a well-developed trajectory of transparency research and extend it. We see inverse transparency as process transparency and disclosure, not as surveillance (Bernstein 2017), as hierarchical control mechanisms are not a part of it. Concerning the design choices for transparency (see Sect. 2.2), the concept is in line with previous studies. Inverse transparency enhances the concept of transparency, with its emphasis on employee empowerment and its connection to leadership approaches.

\section{Discussion and theoretical embedding}

\subsection{Classification of inverse transparency through the lens of control theory}

While agency theory helps in explaining the problem of tensions between employee and manager, control theory helps in developing solutions to overcome them. Interpreting inverse transparency through the lens of control theory sheds light on its innovativeness. According to this theory, control is any measure to align individual behaviors with organizational objectives (Kirsch 1996), thus offering a solution to the agency problem. Control theory originated in the field of management (Ouchi 1977) and has been adapted to the IS context (Cram 2016). It is popular to describe control phenomena in the IS project and IS development context (Cram and Wiener 2020). Work in agile software development teams is often connected to control theory (Fischer et al. 2019). However, the theory's range of application is considered much wider. Researchers suggest that control theory is "not specifically [applicable] to IS project control but to organizational and IS control phenomena more broadly" (Cram and Weiner 2020, p. 72). Therefore, we see great potential in linking our conceptual digital leadership innovation to control theory.

In contrast to agency theory, control theory builds on a new understanding of organizations and human nature (Wiener et al. 2019). Organizations find themselves in the process of blurring borders, continuous innovation, instable ecosystems, a rapidly changing workforce and high knowledge intensity (Wiener et al. 2019). Control theory is designed to meet these characteristics in the digital era. Information asymmetries are not necessarily considered negative but can be helpful in times of growing knowledge intensity. Agency costs should not be addressed following a short-term optimization calculus, but rather a long-term orientation. This may intrinsically motivate employees to act in ways aligned with the firm instead of behaving opportunistically (Wiener et al. 2019). We believe in the 
contemporary relevance of these assumptions. Without building on a redefined understanding of flexible organizations and trustful employees, inverse transparency would not be tangible.

Control theory research can be organized along the lines of who?, what?, when?, why? and how? to identify certain control modes and styles (Cram and Wiener 2020). The concept of inverse transparency embodies a specific configuration of these different control dimensions (see Table 1). Thus, inverse transparency can be classified along the dimensions of control theory. While the dimensions themselves have been defined, their interplay and configurations have not been investigated indepth yet.

The dimension "who?" concerns the control relationship between controller and controllee. It answers the question of which party executes the control. In terms of inverse transparency, we aim to soften the sharp distinction between controller and controllee. Both parties should be empowered to oversee the other independently from hierarchical settings.

The dimension "when?" addresses the control process. Similar to the concept of transparency, control can either focus on the outcome or the process (Kirsch 2004). In contrast to established control processes, inverse transparency embraces both stakeholders throughout the entire process, thus avoiding power asymmetries. Ideally, transparency is always present during every process, in line with the idea of inverse transparency.

Third, control can be introduced for value creation or value appropriation (why?). Value creation refers to the goal to coordinate and empower employees so that they can enhance their capabilities and innovate the current business model (Wiener et al. 2019). Value appropriation represents the scenario described in section three, in the old business model where control is used to track employees and thereby reduce agency risks (Wiener et al. 2019). With inverse transparency, we clearly highlight value creation as employees should actively optimize their processes and think outside the box.

Fourth, "what?" constitutes formal and informal control modes (Cram and Wiener 2020). Formal control mechanisms include standardized processes and activities used to execute control, such as weekly reports. Informal control mechanisms are interpersonal mechanisms that cannot be observed, such as shared

Table 1 Classification of inverse transparency along the dimensions of control theory (adapted from Cram and Wiener 2020)

\begin{tabular}{lll}
\hline Concept & Subconcepts & \\
\cline { 2 - 3 } Control relationship (who) & Controller & Controllee \\
\hline Control process (when) & Process & Outcome \\
Control purpose (why) & Value appropriation & Value co-creation \\
Control modes (what) & Formal & Informal \\
Control style (how) & Coercive & Enabling
\end{tabular}


values and beliefs (Cram and Wiener 2020; Ouchi 1977). Inverse transparency relies on an informal control style that is established naturally between manager and employee without a formalized process. Ideally, the concept would be a shared organizational value.

Last but not least, two different control styles (how?) can be identified (Heuman et al. 2015). On the one hand, control can be introduced in a coercive way as rule enforcement in which the controller is an authoritarian leader. But control can also be executed through an enabling leadership style that empowers employees to better master their daily tasks (Heuman et al. 2015). The latter control style is necessary for effective digital leadership innovations based on inverse transparency.

In sum, inverse transparency is compatible with control theory. Certain specifications of the dimensions of control constitute our understanding of digital leadership innovation, and inverse transparency is characterized by a specific configuration of the control dimensions. Previous work on control theory suggests the need to further investigate the negative consequences, ethical questions and contextual factors for each control configuration (Cram and Wiener 2020). We address this call for research by focusing on the critical reflection of one specific control configuration. Following control theory, we are able to point out potential solutions for the problem of agency.

\subsection{Potentials and challenges of inverse transparency}

Inverse transparency is an opportunity to decrease the tensions exposed by agency theory. As information asymmetries between employees and managers decrease, new forms of control can be established. If digital leadership innovation is based on inverse transparency, managers will refrain from applying hierarchical leadership approaches. If each individual in an organization is well-informed about all processes, managers will not need to apply monitoring mechanisms. Furthermore, employees will not have to invest in signaling activities, as the processes are already visible to everyone. Trust is established among all parties, and transparency is mainly seen as positive. Therefore, the concept can serve as a foundation for digital leadership innovations. We see inverse transparency as a paradigm that influences the foundations of organizations and leadership.

Conversely, inverse transparency has certain requirements and challenges. One crucial requirement is that an organization's culture needs to match the values inherent in the concept. If employees are blamed for mistakes, if decisions are implemented top-down and if innovation and change is only driven by management, trust will be absent. Second, next to a company's culture, certain requirements regarding leadership have to be met. While leader-focused concepts do not align with the principles of inverse transparency, employee-centric and participative leadership styles are a promising beginning. If employees are afraid of an autocratic manager, they are less likely to share their data freely. However, if managers develop and empower their teams, employees will be more cooperative. Third, data must be processed and aggregated in a comprehensive way to avoid information overload. Data must be structured and transformed into information using 
suitable information technologies. Lastly, data collection methods need to be legal, and company agreements have to be considered carefully.

In sum, we find evidence for inverse transparency dissolving the tension between managers and employees. The concept provides a stepping-stone for digital leadership innovations. A fit between the implemented level of transparency and an organization's leadership creates mutually shared values.

\subsection{Implementation of digital leadership innovation based on inverse transparency}

Having conceptualized inverse transparency as a basis for digital leadership innovation, the implementation of the concept is of great practical and theoretical interest. Due to the nature of this conceptual work, it is not our aim to provide empirical evidence for successful implementation processes. Instead, in the following section, we build on the well-established IS implementation model to derive propositions.

The implementation of digital innovation implies to "install and maintain IS from both a technical and an organizational perspective, including new governance systems, training, and processes" (Kohli and Melville 2018, p. 203). This organizational change is considered a complex, nonlinear process which can be depicted by the IS implementation model (Cooper and Zmud 1990). The model has been applied in various settings to describe the diffusion of technological innovations in companies, such as ERP (Cooper and Zmud 1990), cloud computing (Conboy and Morgan 2012) or big data applications (Weibl and Hess 2018). Considering novel leadership approaches, combined with transparency-enabling IT artifacts, as digital innovation, the framework is suitable to describe the implementation process along six phases.

The different phases of the model by Cooper and Zmud (1990) can be adapted to the context of digital leadership innovation based on inverse transparency, leading to a normative implementation process (see Fig. 5):

In the initiation phase, difficulties regarding the current leadership approach are detected and pressure for change evolves. Following the above-described tensions for managers and employees from agency theory, this pressure can be driven topdown or bottom-up, as both parties benefit from novel leadership approaches. While searching for a solution for the detected problem, a match is found between a digital leadership innovation based on inverse transparency and its intended application in the organization. Thus, we propose:

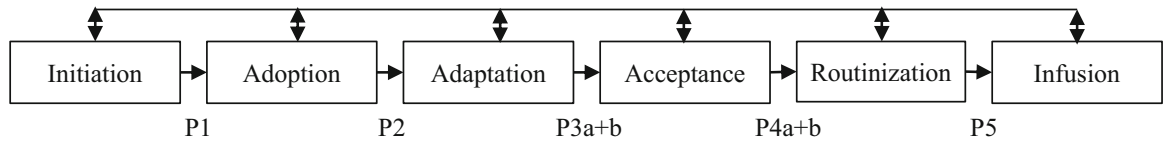

Fig. 5 IS implementation process for digital leadership innovtaion based on inverse transparency following Cooper and Zmud (1990) 
P1 The initiation of digital leadership innovation based on inverse transparency is driven by dissatisfaction with current leadership practices and the desire for increased data transparency inside an organization.

In the adoption phase, organizational negotiation processes ensue and the different organizational stakeholders define the optimal level of transparency as well as suitable framing conditions. The negotiation is impacted by the current leadership style, the organization's culture and the current availability and use of data. Finally, the decision is made to adopt a digital leadership innovation that is based on inverse transparency in the organization. We derive:

P2 The adoption of digital leadership innovation based on inverse transparency is a negotiation process and the optimal level of transparency depends on the individual organization. A high level of data availability leads to a higher optimal level of inverse transparency.

In the adaptation phase, technical and organizational framing conditions are adjusted and the digital leadership innovation is implemented in the organization. A novel innovation culture and adjusted leadership values, as well as a suitable IT environment, support the adaptation. Inverse transparency requires a high availability of employee data. Thus IS that collect and analyze leadership-related data are connected and organizations avoid media disruptions between these systems. Furthermore, organizational members are trained to employ the digital innovation, which means they gain competence in assessing and using relevant data. To describe this mutual interaction, we propose:

P3a To adapt digital leadership innovation based on inverse transparency successfully, organizations are required to develop a suitable innovation and error culture.

P3b Reversely, the adaptation of the digital leadership innovation shapes organizations' innovation and error culture.

Next, organizational members commit to the usage of the digital leadership innovation based on inverse transparency by initially applying it in the acceptance phase. Every stakeholder owns the competence and the willingness to use novel IS that provide transparency. The acceptance of the IT artifact will successively lead to the acceptance of the organizational artifact. We suggest:

$\mathrm{P} 4 \mathrm{a}$ The acceptance of digital leadership innovation based on inverse transparency facilitates employee empowerment and leads to an increase in employee satisfaction.

P4b The acceptance of digital leadership innovation based on inverse transparency drives enabling control styles and leads to diversification of power distribution.

In the routinization phase, the novel leadership approach is routinely used inside the firm. Leadership is based on enabling control styles that empower employees. Novel performance measures, e.g. $360^{\circ}$ feedback, are implemented that do not 
promote tracking control styles. Thus, transparency inside the firm increases and agency costs can be reduced. Finally, we derive:

P5 The routinization of digital leadership innovation based on inverse transparency facilitates the availability of data to diverse groups of stakeholders and leads to a reduction of firms' overall agency costs.

Lastly, in the infusion phase, the digital leadership innovation is not only applied in a routinely fashion, but also in a sophisticated manner within the organization and has positive impacts on the whole firm. This phase can only be reached after several years and multiple iterations of the process as the concept incorporates profound strategical challenges. Each organization might undergo a different implementation process along the six phases and we strongly recommend investigating this phenomenon with empirical data.

\section{Conclusion and implications for further research}

\subsection{Summary}

In our article, we position digital leadership innovations as a new supportive type of digital innovations. The two artifacts of digital innovations, the technical side consisting of an IT artifact and the innovative digital business concept addressing the organizational dimension, are present. Following this line of argumentation, leadership is not only an enabler of distinct digital innovations but the core of digital innovations.

Digital leadership innovations are driven by the availability of novel digital technologies that lead to innovative digital solutions. In the case of digital leadership innovations, the IT artifact is represented by a growing amount of data on employees and specialized leadership-related IS that generate value from the data. As organizational requirements are changing and employees strive for empowerment, these technologies spread rapidly within firms. This development calls into question the usefulness of former management and leadership practices.

The growing amount of data on employees has changed the way work is carried out. On the one hand, data makes it possible for managers to observe employees' work at little cost in real time, which can potentially lead to control-driven, autocratic leadership concepts. On the other hand, data can be used to empower employees and to apply employee-centric leadership concepts. Managers face a complex challenge in integrating data into their leadership styles, and depending on how they integrate data, employees either benefit or suffer from transparency. The promising benefits of transparency are in tension with the perceived risk of lacking privacy.

The concept of inverse transparency aims to bridge these tensions by expanding and re-interpreting the traditional understanding of transparency. Inverse transparency is based on the principles of making data collection and data usage visible, engaging employees in data protection mechanisms and empowering them to use available data for process improvements. As inverse transparency offers incentives 
for both employees and managers, privacy concerns are addressed, data are shared more openly, new forms of control are established and employees are engaged and empowered. Inverse transparency addresses the organizational artifact of digital innovations. It serves as a valuable basis for employee-centric, data-enabled leadership approaches in a digital working environment.

\subsection{Contributions}

Our conceptual work is of an explanatory theoretical nature (Gregor 2006). It contributes in the main to the theoretical body of knowledge about transparency. First, we revise established types of digital innovations with a new, supportive type of digital leadership innovation. Considering leadership as central to digital innovations establishes new horizons for research on digital leadership approaches. Leadership can be game changing and much more than an enabler in a firm's digital transformation. With this new understanding, we stress the value of digital leadership innovations as a success factor.

Second, we extend the earlier view on transparency with our conceptualization of inverse transparency. Established understandings of transparency depict it as a onedimensional relationship between manager and employee. However, transparency should not only be viewed as monitoring or surveillance of employees but rather as a chance to empower them and strengthen their self-organization. By redefining the concept of transparency, new ways of control and leadership can be investigated.

Third, our concept of inverse transparency employs agency and control theory in insightful, new ways. Agency theory assists in explaining the origin of information asymmetries between managers and employees and the resulting tensions. Analyzing transparency through the lens of control theory suggests solutions for the trade-off between positive and negative effects of transparency. Inverse transparency allows for new forms of control that empower employees. As daily processes in firms become more transparent, employees can evaluate the outcome of their actions more precisely and are motivated to contribute constructively.

Fourth, we point out the implementation process for digital leadership innovations based on inverse transparency from a normative point of view. By illustrating the different stages of the implementation and by deriving propositions in a qualitative way, we highlight the implementation and assimilation of inverse transparency in organizations. The applicability of the IS implementation model for this novel type of digital innovation is illustrated and serves as a stepping-stone for further research.

Lastly, we respond to the call for a holistic analysis that combines IS and management literature on leadership in the digital age (Cortellazzo et al. 2019). While research on the influence of digitalization on leadership is mostly conducted independently in these two fields, we take valuable findings from both into account and enhance the findings of previous research.

From a practical perspective, our research addresses a highly relevant topic. Independent from a company's size, its industry or location, firms face challenges in integrating data sustainably in leadership. We provide guidance on how to manage the rising tensions as data becomes increasingly available along a process model. 
Though we are not yet able to derive concrete recommendations, our study offers new perspectives for leaders, which needs to be investigated further.

\subsection{Limitations}

Though thoroughly researched, our study has certain limitations. To date, digital leadership innovations based on inverse transparency have been conceptualized but not been validated in practice. We focus on the theory-driven conceptualization of inverse transparency as the basis for new leadership approaches. Research on the design, implementation and success of digital leadership innovations is needed. Both qualitative and quantitative empirical studies are essential to better understand this area of research.

Moreover, we aimed to provide a holistic overview about existing research streams without analyzing each stream in-depth. We evaluated the concept in the light of different theories. Thus, our research covers a broad scope but lacks domain-specific details.

In addition to these methodological limitations, the concept of inverse transparency itself contains certain limitations. Researchers have pointed out the limits of transparency as its implementation can be costly, time-consuming or even impossible because of discrepancies between assumptions and reality (Valentinov et al. 2019). Bernstein describes this transparency paradox: "broad visibility, intended to increase transparency, can breed hiding behavior and myths of learning and control, thereby reducing transparency" (Bernstein 2012, p. 209). Inefficiencies can arise when too much data are available. Furthermore, transparency is intended to provide incentives for all stakeholders, but managers might not be willing to establish full transparency if it means their information advantage diminishes. Taking inverse transparency to the extreme, hierarchies are made obsolete and employees can manage themselves. This contradicts the current organizational structure of most companies.

In our study of inverse transparency, we do not discuss potential benefits for employees when strict control mechanisms are implemented, but we assume that this is undesirable for the employee. In contrast to our suggestion of control leading to mistrust, it could be seen as an opportunity for employees to use it as selfprotection, for example, by documenting hours worked. Finally, inverse transparency is not a normative solution but a negotiation process. This process will differ among organizations and calls for further investigation. With regard to these limitations, we suggest the following agenda for further research.

\subsection{Agenda for further research}

\subsubsection{Design of IS for inverse transparency}

Digital leadership innovations based on the concept of inverse transparency can only be implemented in companies if IS are suitable to support its implementation. Thus, the adaptation of existing tools and the development of new systems is essential. Previous scholarship has highlighted different design choices for 
transparency-enabling IS. The understanding of transparency (Bernstein 2017), the type of data and the frequency of observation (Schnackenberg and Tomlinson 2016) are among the important design criteria. These design features should be considered when developing systems that facilitate inverse transparency. Scholarly contributions from the field of system engineering could shed light on the design of these tools.

\subsubsection{Process view on inverse transparency}

The implementation of inverse transparency is outlined from a conceptual point of view in this study, leaving room for multiple in-depth studies. First, it would be valuable to analyze the negotiation process in more detail. Applying formal modeling techniques using agency theory could offer new insights. Second, the different phases of the IS implementation process should be investigated with empirical data and the derived propositions should be tested. A potential outcome could be a process model illustrating different milestones for the implementation of inverse transparency.

\subsubsection{Empirical validation}

We have only introduced inverse transparency on a conceptual level. However, we highly value its evaluation. We consider design science a suitable method to combine both practical and theoretical perspectives, as it uses both the practiceoriented rigor cycle and the theory-oriented rigor cycle (Hevner et al. 2004). Research on digital innovations and design science research articulate well (Hevner et al. 2019). Thus, developing the concept of inverse transparency further and implementing it using design science research would help in the empirical evaluation of the concept. Furthermore, inverse transparency needs to be operationalized, and studies of both organizational transparency (Awad and Krishnan 2006) and data transparency (Byrd and Turner 2000) can provide guidance.

\subsubsection{Inverse transparency and users' acceptance}

Digital leadership innovation needs to be accepted by all stakeholders to be implemented successfully in organizations. Contributions on technology acceptance can serve as a stepping-stone for research in this field (Lee et al. 2003). In contrast to the individual decision that is observed in the technology acceptance model, employees are not completely free in their decision to adopt digital innovation. This adds complexity to established research on acceptance.

\subsubsection{Linking the concept to privacy research}

From our perspective, transparency and privacy research are closely connected. The well-established privacy paradox (Awad and Krishnan 2006) can be transferred to the transparency paradox (Bernstein 2012). The perspective of research changes: 
While customers and their privacy decisions are the subject of privacy research, we assume similar mechanisms for employees and their privacy decisions. Transparency can counterbalance privacy concerns and thus address privacy issues in the workplace.

\subsubsection{Ethical and legal considerations}

Ethical and legal considerations set the framing conditions for inverse transparency. Distinct organizational cultures lead to different perceptions of transparency (Leidner and Kayworth 2006). In addition, legal regulations about data protection vary among countries and firms. Practices that have successfully been established in certain countries would fail to pass workers' councils in firms in other countries. Investigating these cultural and legal differences, for example, in qualitative case studies with multiple globally distributed departments of a company, would offer insight into an evaluation of the generalizability of the concept.

\subsubsection{Linking transparency to the field of HRM}

As research suggests, research on innovative leadership approaches should take digital technologies into account (Cortellazzo et al. 2019). Next to traditional leadership concepts, interdisciplinary work on new approaches is highly valued. Thus, we recommend connecting the digital leadership innovation that is based on inverse transparency to HRM. Further work should be conducted on the changing roles of managers and the relationship between managers and employees. Furthermore, stressing analogies to existing leadership concepts would be of great importance. Modern leadership approaches like e-leadership or shared leadership could offer points of reference for comparison.

Funding Research project funded by Bundesministerium für Bildung und Forschung.

Availability of data and material (data transparency) Not applicable.

Code availability (software application or custom code) Not applicable.

\section{Compliance with ethical standards}

Conflict of interest Not applicable.

Open Access This article is licensed under a Creative Commons Attribution 4.0 International License, which permits use, sharing, adaptation, distribution and reproduction in any medium or format, as long as you give appropriate credit to the original author(s) and the source, provide a link to the Creative Commons licence, and indicate if changes were made. The images or other third party material in this article are included in the article's Creative Commons licence, unless indicated otherwise in a credit line to the material. If material is not included in the article's Creative Commons licence and your intended use is not permitted by statutory regulation or exceeds the permitted use, you will need to obtain permission directly from the copyright holder. To view a copy of this licence, visit http:// creativecommons.org/licenses/by/4.0/. 


\section{References}

Abrahamsson, Pekka, Kieran Conboy, and Xiaofeng Wang. 2017. "Lots done, more to do": The current state of agile systems development research. European Journal of Information Systems 18 (4): 281-284.

Albu, Oana Brindusa, and Mikkel Flyverbom. 2019. Organizational transparency: Conceptualizations, conditions, and consequences. Business and Society 58 (2): 268-297.

Alder, G. Stoney. 2001. Employee reactions to electronic performance monitoring: A consequence of organizational culture. The Journal of High Technology Management Research 12 (2): 323-342.

Aral, Sinan, Erik Brynjolfsson, and Wu. Lynn. 2012. Three-way complementarities: Performance pay, human resource analytics, and information technology. Management Science 58 (5): 913-931.

Aung, Myo Min, and Yoon Seok Chang. 2014. Traceability in a food supply chain: Safety and quality perspectives. Food Control 39: 172-184.

Avolio, B.J., J.J. Sosik, S.S. Kahai, and B. Baker. 2014. E-leadership: Reexamining transformations in leadership source and transmission. Leadership Quarterly 25: 105-131.

Avolio, Bruce J., Surinder Kahai, and George E. Dodge. 2000. E-leadership. The Leadership Quarterly 11 (4): 615-668.

Awad, Naveen Farag, and M.S. Krishnan. 2006. The personalization privacy paradox: An empirical evaluation of information transparency and the willingness to be profiled online for personalization. MIS Quarterly 30 (1): 13-28.

Backhaus, Nils. 2019. Kontextsensitive Assistenzsysteme und Überwachung am Arbeitsplatz: Ein metaanalytisches Review zur Auswirkung elektronischer Überwachung auf Beschäftigte. Zeitschrift für Arbeitswissenschaft 73: 2-22.

Beare, H., B. Caldwell, and R. Millikan. 1997. Dimensions of leadership. In Leadership and teams in educational management. Leadership and management in education, ed. Megan Crawford, Lesley Kydd, and Colin R. Riches, 24-39. Buckingham: Open Univ. Press.

Berglund, T. 2014. Corporate governance and optimal transparency. In The Oxford handbook of economic and institutional transparency, 359-371. Oxford: Oxford University Press.

Bernstein, Ethan. 2012. The transparency paradox: A role for privacy in organizational learning and operational control. Administrative Science Quarterly 57 (2): 181-216.

Bernstein, Ethan. 2017. Making transparency transparent: The evolution of observation in management theory. Academy of Management Annals 11 (1): 217-266.

Bharadwaj, Anandhi, Omar El Sawy, Paul Pavlou, and N. Venkatraman. 2013. Digital business strategy: Towards a next generation of insights. MIS Quarterly 37 (2): 471-482.

Boes, Andreas. 2020. Inverse transparenz. https://www.inversetransparenz.de/. Accessed 07.02.2020.

Boudreau, John, and Wayne Cascio. 2017. Human capital analytics: Why are we not there? Journal of Organizational Effectiveness: People and Performance 4 (2): 119-126.

Brin, D. 1998. The transparent society. Will technology force us to choose between privacy and freedom? New York: Basic Books.

Byrd, Terry Anthony, and Douglas E. Turner. 2000. Measuring the Flexibility Of Information Technology Infrastructure: Exploratory analysis of a construct. Journal of Management Information Systems 17: 167-208.

Carasco-Saul, Marie, Woocheol Kim, and Taesung Kim. 2015. Leadership and employee engagement: Proposing research agendas through a review of literature. Human Resource Development Review 14 (1): 38-63.

Châlons, Christophe, and Nicole Dufft. 2017. The role of IT as an enabler of digital transformation. In The drivers of digital transformation, ed. Ferri Abolhassan. Berlin: Springer.

Christensen, L.T., and G. Cheney. 2015. Peering into transparency: Challenging ideals, proxies, and organizational practices. Communication Theory 25: 70-90.

Conboy, K., and L. Morgan. 2012. Assimilation of the cloud: Challenges to acceptance, routinisation and infusion of cloud computing. In International Conference on Information Systems. Orlando.

Cooper, Randolp B., and Robert W. Zmud. 1990. Information technology implementation research: A technological diffusion approach. Management Science 36 (2): 123-139.

Cortellazzo, Laura, Elena Bruni, and Rita Zampieri. 2019. The role of leadership in a digitized world: A review. Frontiers in Psychology 10: 1-21.

Cram, W.A. 2016. Information systems control: A review and framework for emerging information systems processes. Journal of the Association for Inofrmation Systems 17 (4): 216-266. 
Cram, W.A., and Martin Wiener. 2020. Technology-mediated control: Case examples and research directions for the future of organizational control. Communications of the Association for Information Systems 46 (4): 70-91.

de Camargo Fiorini, Paula, Bruno Michel Roman Pais. Seles, Charbel Jose Chiappetta. Jabbour, Enzo Barberio Mariano, and Ana Beatriz Lopes. de Sousa Jabbour. 2018. Management theory and big data literature: From a review to a research agenda. International Journal of Information Management 43: 112-129.

Diamante, Thomas, and Manuel London. 2002. Expansive leadership in the age of digital technology. Journal of Management Development 21: 404-416.

Dinh, Jessica E., Robert G. Lord, William L. Gardner, Jeremy D. Meuser, Robert C. Liden, and Hu. Jinyu. 2014. Leadership theory and research in the new millennium: Current theoretical trends and changing perspectives. The Leadership Quarterly 25 (1): 36-62.

Edmunds, Angela, and Anne Morris. 2000. The problem of information overload in business organisations: A review of the literature. International Journal of Information Management 20 (1): 17-28.

Eisenhardt, Kathleen M. 1989. Agency theory: An assessment and review. Academy of Management Review 14 (1): 57-74.

Fischer, T., Hevner, A., and Riedl, R. 2019. Control portfolio adaptation in scrum: Initial findings from a practitioner survey. In International Conference on Information Systems. Munich.

Gal, Ulrich, Tina Blegind Jensen, and Mari-Klara Stein. 2017. People analytics in the age of big data: An agenda for IS research. In Proceedings of the Annual International Conference on Information Systems. Atlanta: Association for Information Systems.

Gerlach, Jin, Thomas Widjaja, and Peter Buxmann. 2015. Handle with care: How online social network providers' privacy policies impact users' information sharing behavior. Journal of Strategic Information System 24 (1): 33-43.

Gerth, A.B., and J. Peppard. 2016. The dynamics of CIO derailment: How CIOs come undone and how to avoid it. Business Horizons 59: 61-70.

Gierlich, Maren, and Thomas Hess. 2020. Towards an understanding on datas' influence on leadership. In Internationale Tagung der Wirtschaftsinformatik. Potsdam.

Granados, Nelson, and Alok Gupta. 2013. Transparency strategy: Competing with information in a digital world. MIS Quarterly 37 (2): 637-641.

Gregor, Shirley. 2006. The nature of theory in information systems. MIS Quarterly 30 (3): 611-642.

Haddud, Abubaker, and Dorothy McAllen. 2018. Digital workplace management: Exploring aspects related to culture, innovation, and leadership. Paper presented at the Portland International Conference on Management of Engineering and Technology.

Haffke, Ingmar, Bradley Kalgovas, and Alexander Benlian. 2016. The role of the CIO and the CDO in an organization's digital transformation. Paper presented at the International Conference on Information Systems, Dublin.

Hambley, Laura A., Thomas A. O’Neill, J.B. Theresa, and Kline. . 2007. Virtual team leadership: The effects of leadership style and communication medium on team interaction styles and outcomes. Organizational Behavior and Human Decision Processes 103: 1-20.

Hays, ZukunftsAllianz Arbeit \& Gesellschaft e. V., Gesellschaft für Wissensmanagement e. V., and TU München. 2017. Der Ruf nach Freiheit: Innovationsförderliche Arbeitswelten aus Sicht der Arbeitenden. $\quad$ https://www.hays.de/documents/10192/118775/studie-freiheit-sicherheit.pdf/ 979efc80-ca49-4d85-a7bc-aeb1f3592ee6. Accessed 30 Sep 2019.

Heald, D. 2006. Varieties of transparency. In Transparency: The key to better governance?, ed. C. Hood and D. Heald, 25-43. Oxford: Oxford University Press.

Henfridsson, Ola, and Bendik Bygstad. 2013. The generative mechanisms of digital infrastructure evolution. MIS Quarterly 37 (3): 907-931.

Henttonen, Katja, Pasi Pussinen, and Timo Koivumäki. 2012. Managerial perspective on open source collaboration and networked innovation. Journal of Technology Management and Innovation 7: $135-147$.

Hess, Thomas. 2002. Informations-und Kommunikationstechnologien als Treiber neuer ControllingLösungen-Skizze eines Forschungsprogramms. In Controlling als akademische Disziplin. Schriften des Center for Controlling and Management (CCM), ed. J. Weber and B. Hirsch. Wiesbaden: Deutscher Universitätsverlag.

Heuman, J., M. Wiener, U. Remus, and M. Mähring. 2015. To coerce or to enable? Exercising formal control in a large information systems project. Journal of Information Technology 30: 337-351. 
Hevner, Alan, Salvatore March, Jinsoo Park, and Sudha Ram. 2004. Design science in information systems research. MIS Quarterly 28 (1): 75-105.

Hevner, Alan, Jan vom Brocke, and Alexander Mädche. 2019. Roles of digital innovation in design science research. Business and Information Systems Engineering 61 (1): 3-8.

Hinings, Bob, Thomas Gegenhuber, and Royston Greenwood. 2018. Digital innovation and transformation: An institutional perspective. Information and Organization 28: 52-61.

Hofmann, Yvette. 2007. Transparenz in Unternehmen: Leistungsanreiz oder Leistungsbremse für ihre Mitglieder? Zeitschrift für Management 2 (1): 6-27.

Johnson, Richard D., Kimberly M. Lukaszewski, and Dianna L. Stone. 2016. The evolution of the field of human resource information systems: Co-evolution of technology and HR processes. Communications of the ACM 38: 533-553.

Johnson, Richard D., Kimberly M. Lukaszewski, and Dianna L. Stone. 2016. The evolution of the field of human resource information systems: Co-evolution of technology and HR processes. Communications of the Association for Information Systems 38: 533-553.

Kavanagh, Michael J., and Richard David Johnson. 2018. Human resource information systems: Basics, applications, and future directions. Los Angeles: Sage.

Khatri, Vijay, and Binny M. Samuel. 2019. Analytics for managerial work. Communications of the ACM 62 (4): 100-128.

Kirsch, L.J. 1996. The management of complex tasks in organizations: Controlling the systems development process. Organizational Science 7 (1): 1-21.

Kirsch, L.J. 2004. Deploying common systems globally: The dynamics of control. Information Systems Research 15 (4): 374-395.

Kohli, Rajiv, and Nigel P. Melville. 2018. Digital innovation: A review and synthesis. Information Systems Journal 29: 200-223.

Korica, M., D. Nicolini, and B. Johnson. 2015. In Search of 'Managerial Work': Past, present and future of an analytical category. International Journal of Management Reviews 19: 151-174.

Lang, Rainhart, and Irma Rybnikova. 2014. Aktuelle Führungstheorien und -konzepte. Wiesbaden: Springer Fachmedien.

Lee, Younghwa, Kenneth A. Kozar, and Kai R. T. Larsen. 2003. The technology acceptance model: Past, present, and future. Communications of the ACM 12 (50): 752-780.

Leidner, Dorothy E., and Timothy Kayworth. 2006. Review: A review of culture in information systems research: Toward a theory of information technology culture conflict. MIS Quarterly 30 (2): 357-399.

Lim, Sanghee, Terence J.V.. Saldanha, Suresh Malladi, and Nigel P. Melville. 2013. Theories used in information systems research: Insights from complex network analysis. Journal of Information Technology Theory and Application 14 (2): 5-46.

Maier, Christian, Sven Laumer, Andreas Eckhardt, and Tim Weitzel. 2013. Analyzing the impact of HRIS implementations on HR personnel's job satisfaction and turnover intention. The Journal of Strategic Information Systems 22 (3): 193-207.

Matt, Christian, Thomas Hess, and Alexander Benlian. 2015. Digital transformation strategies. Business and Information Systems Engineering 57 (5): 339-343.

Mintzberg, Henry. 2007. The manager's jobs: Folklore and fact. In Leadership, ed. R.P. Vecchio, 49-61. Notre Dame: University of Notre Dame Press.

Mittal, Sameer, Muztoba Khan, David Romero Ahmad, and Thorsten Wuest. 2019. Smart manufacturing: Characteristics, technologies and enabling factors. Journal of Engineering Manufacture 233 (5): 1342-1361.

Nambisan, Satish, Kalle Lyytinen, Ann Majchrzak, and Michael Song. 2017. Digital innovation management: Reinventing innovation management research in a digital world. MIS Quarterly 41 (1): 215-226.

Nerur, Sridhar, Radha Kanta Mahapatra, and George Mangalaraj. 2005. Challenges of migrating to agile methodologies. Communications of the ACM 48 (5): 72-78.

Nussbaumer, Philipp, and Gerhard Schwabe. 2012. "Enforced" vs. "Casual" transparency-Findings from IT-supported financial advisory encounters. ACM Transactions on Management Information Systems 3 (2): 1-19.

Ouchi, W.G. 1977. The relationship between organizational structure and organizational control. Administrative Science Quarterly 22 (1): 95-113. 
Parviainen, Päivi., Maarit Tihinen, Jukka Kääriäinen, and Susanna Teppola. 2017. Tackling the digitalization challenge: How to benefit from digitalization in practice. International Journal of Information Systems and Project Management 5 (1): 63-77.

Picot, Arnold. 1989. Wechselwirkungen zwischen Allgemeiner Betriebswirtschaftslehre und neueren Speziellen Betriebswirtschaftslehren. In Die Betriebswirtschaftslehre im Spannungsfeld zwischen Generalisierung und Spezialisierung, ed. Werner Kirsch and Arnold Picot, 361-379. Wiesbaden: Gabler $\mathrm{GmbH}$.

Pierce, L., D. Snow, and A. McAfee. 2015. Cleaning house: The impact of information technology monitoring on employee theft and productivity. Management Science 61 (10): 2299-2319.

Rodríguez-Mazahua, Lisbeth, Cristian-Aarón. Rodríguez-Enríquez, José Luis. Sánchez-Cervantes, Jair Cervantes, Jorge Luis García-Alcaraz, and Giner Alor-Hernández. 2016. A general perspective of big data: Applications, tools, challenges and trends. Journal of Supercomputing 72 (8): 3073-3113.

Schnackenberg, A., and E. Tomlinson. 2016. Organizational transparency: A new perspective on managing trust in organization-stakeholder relationships. Journal of Management 42: 1784-1810.

Schwarzmüller, Tanja, Prisca Brosi, Denis Duman, and Isabell M. Welpe. 2018. How does the digital transformation affect organizations? Key themes of change in work design and leadership. Management Review 29: 114-138.

Sebastian, Ina M., Jeanne W. Ross, Cynthia Beath, Martin Mocker, Kate G. Moloney, and Nils O. Fonstad. 2017. How big old companies navigate digital transformation. MIS Quarterly Executive 16 (3): 197-213.

Stanton, Jeffrey M., and Elizabeth M. Weiss. 2000. Electronic monitoring in their own words: An exploratory study of employees' experiences with new types of surveillance. Coputers in Human Behavior 16 (4): 423-440.

Tumbas, Sanja, Nicholas Berente, and Jan vom Brocke. 2018. Digital innovation and institutional entrepreneurship: Chief digital officer perspectives of their emerging role. Journal of Information Technology 33 (2): 188-202.

Tursunbayeva, Aizhan, Stefano Di Lauro, and Claudia Pagliari. 2018. People analytics-A scoping review of conceptual boundaries and value propositions. International Journal of Information Management 43: 224-247.

Valentinov, Vladislav, Gert Verschragenen, and Kristof Van Assche. 2019. The limits of transparency: A systems theory view. Systems Research and Behavioral Science 36: 289-300.

Vial, Gregory. 2019. Understanding digital transformation: A review and a research agenda. Journal of Strategic Information Systems 28: 118-144.

Wamba, Samuel Fosso, Shahriar Akter, Andrew Edwards, Geoffrey Chopin, and Denis Gnanzou. 2015. How 'big data' can make big impact: Findings from a systematic review and a longitudinal case study. International Journal of Production Economics 165: 234-246.

Weibl, Johannes, and Thomas Hess. 2018. Success or failure of big data: Insights of managerial challenges from a technology assimilation perspective. In Multikonferenz Wirtschaftsinformatik 2018. Lüneburg.

Wiener, M., M. Mähring, U. Remus, C. Saunders, and A.W. Cram. 2019. Moving IS project control research into the digital era: The "Why" of control and the concept of control purpose. Information Systems Research 30 (4): 1387-1401.

Wiesböck, Florian, and Thomas Hess. 2020. Digital innovations-Embedding in organizations. Electronic Markets 30: 75-86.

Yukl, Gary. 2013. Leadership in organizations. Upper Saddle River: Pearson.

Publisher's Note Springer Nature remains neutral with regard to jurisdictional claims in published maps and institutional affiliations. 